Recepción: 19 / 01 / 2017

Aceptación: 11 / 05 / 2017

Publicación: 15 / 07 / 2017

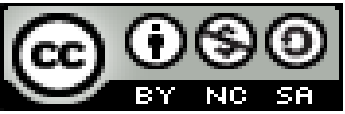

Ciencias económicas y empresariales

Artículo de investigación

\title{
Algunas razones para ser mejores gerentes de empresas
}

\author{
Some reasons to become better business managers
}

\section{Algumas razões para se tornar melhores gerentes de negócios}

\author{
Andrea L. Ruiz-Vélez ${ }^{\mathrm{I}}$ \\ alruiz@sangregorio.edu.ec \\ María E. Arteaga-García II \\ mearteaga@sangregorio.edu.ec \\ Christian A. Vera-Álava III \\ cavera@sangregorio.edu.ec
}

Correspondencia: alruiz@sangregorio.edu.ec

\footnotetext{
I Magister en Gestión Empresarial, Ingeniera en Comercio Exterior, Docente de la Universidad San Gregorio de Portoviejo, Portoviejo, Ecuador.

II Magister en Tributación y Finanzas, Diploma Superior en Tributación, Especialista en Tributación, Economista, Docente de la Universidad San Gregorio de Portoviejo, Portoviejo, Ecuador.

III Magister en Tributación y Finanzas, Diploma Superior en Tributación, Economista, Docente de la Universidad San Gregorio de Portoviejo, Portoviejo, Ecuador.
} 
- Las organizaciones persiguen metas compartidas.

- Las organizaciones están formadas por partes y éstas interaccionan entre sí para alcanzar los objetivos.

- Las organizaciones son un arreglo sistemático de personas encaminadas a realizar un propósito específico.

- Las organizaciones comparten tres características en común: propósito definido, personal y estructura sistemática.

Es importante considerar una organización como un sistema socio-técnico en el cual el gerente debe administrar recursos de: (USACA, s/f)

- Personal.

- Tareas

- Tecnología.

- Cultura.

- Estructura.

Para administrar estos recursos, todos los gerentes necesariamente deben tener las capacidades o las habilidades en las funciones gerenciales. Es decir, al llevar a cabo sus funciones de planeación, organización, dirección y control los gerentes deben obtener ciertos resultados. Es probable que estos resultados sean superiores a lo que se podrían lograr si los gerentes añadieran a sus operaciones otros ingredientes.

Este trabajo pretende exponer los ingredientes extras que debe añadirle un gerente a su cargo para obtener resultados superiores.

Para ello se utiliza el análisis documental.

\section{Desarrollo}

El gerente, es el individuo de una organización que dirige las actividades de otro. Los gerentes tienen una amplia variedad de títulos: los gerentes de primera línea, gerentes intermedios y los gerentes de alta dirección (Robbins, 1992). 
Un cierto modelo gerencial dominó el campo de la gerencia durante las últimas décadas, con ajustes menores en sus propuestas básicas. Produjo sus resultados, pero claramente su ciclo parece agotado.

Las dificultades del modelo gerencial tradicional, han determinado su profunda crisis actual y surge la necesidad de un reemplazo integral del modelo y del desenvolvimiento de un nuevo paradigma gerencial.

Existen propiedades básicas que cualquier gerente debe tener como: orden personal, voz de mando, conocimiento del sector, formación académica, sentimiento de superación desarrollado, etc. Es decir que básicamente las características en el futuro para el gerente serán las mismas que se han considerado desde los comienzos de la ciencia y tecnología administrativa de la teoría moderna u orgánica del siglo $\mathrm{XX}$, sin embargo si será necesario que este nuevo gerente se preocupe mucho más por mantenerse informado de las nuevas tendencias de la economía de su sector y de las nuevas herramientas que la tecnología fabrica y pone a su alcance, es decir que debe ser consciente de la velocidad del mercado. Aparte de esto se exigirá una formación más rigurosa y profunda ya que la profesionalización de la gerencia es un fenómeno bastante marcado en las economías de mercado (USACA, s/f).

Varios autores coinciden en que los mayores obstáculos para el desenvolvimiento de una gerencia eficiente están en la comunicación al interior y exterior de la empresa y el desconocimiento de los procesos productivos por parte del gerente.

La gerencia del futuro será una gerencia que operará, en medio de parámetros de cambio inéditos, y en una situación básica que pensadores gerenciales avanzados han denominado un "mundo de entrometidos". Una situación en donde la "aldea global", determina que infinidad de actores del contexto, ejerzan influencias sobre cada organización, se "entrometan" en su entorno de decisiones de diversas formas Será en definitiva una gerencia que actuará en medio de la turbulencia. Hay que aprender a gerenciar complejidad (USACA, s/f).

Para que un gerente actúe ante la complejidad, debe comprender que la complejidad misma de la realidad organizacional aparece ante la existencia de un polo lógico y uno empírico, los cuales desencadenan en dificultades lógicas y dificultades empíricas, por lo tanto es preciso investigar los fenómenos desde esas dos perspectivas. Esta racionalidad opta por la complementariedad y la 
conjunción de los conocimientos disciplinarios (sin prescindir de sus especificidades), es decir, toma el camino de la transdisciplinariedad y del holismo. Así mismo, el gerente debe comprender: (a) nuevas lógicas de hacer ciencia; (b) las complejidades en relación a las complejidades internas y externas a la organización (sociedad, ambiente, competencia); (c) la importancia de la construcción de nuevas realidades empresariales, a través de estrategias producto, por una parte, de la participación de los seres que hacen vida en la organización, y por la otra de sus grupos de interés externos, en la búsqueda constante del porvenir y devenir; (d) que en la naturaleza y en la sociedad, entre los humanos y los demás seres vivos proliferan los desórdenes, pero también son creadores de órdenes, dando como resultados fenómenos transcomplejos, donde la relación causa y efecto que éstos puedan tener en el desempeño de las organizaciones se ve limitado a espacios-tiempos relativamente cortos (Pérez M.A., 2014).

Hay una especie de esquema axiomático, nacido originariamente en Henry Fayol, y objeto de ajustes y desagregaciones que no se han apartado del núcleo original. El gerente como lo repiten los manuales del modelo: planifica, dirige, organiza y controla. Debe aprender los instrumentos técnicos para cada una de estas labores y concentrarse en ellas. Al mismo tiempo, en versiones más avanzadas del modelo, deben fluir hacia él diversos sistemas de información y dedicar considerable tiempo a su análisis sistemático (Garrido J.A., 1993).

La imagen que surge como ideal, es la de un gerente concentrado en su oficina, provisto de unidades automatizadas que van arrojando información sobre su escritorio, defensor implacable de su tiempo, dedicado a planificar y pensar, protegido por una línea infranqueable de secretarias que sólo darán entrada a interlocutores de excepcional jerarquía. Sus contactos humanos centrales son con su alto staff inmediato.

Ante las nuevas demandas con relación al entorno en cambio continuo, a la incertidumbre, a la complejidad es necesario un nuevo gerente. Efectivamente una serie de importantes estudios modernos han demostrado que los verdaderos gerentes de excelencia se dedican a otras tareas más allá de las funciones gerenciales.

En la Universidad de Harvard el profesor John Kotter analizó en el campo las actividades concretas de gerentes con altos logros. El equipo de investigadores siguió cuidadosamente 
durante un extenso periodo el día de trabajo de los "exitosos". Verificó que su conducta diaria era del siguiente tipo:

- Pasan más del $75 \%$ de su tiempo conversando con otros.

- Sus interlocutores son de una gama muy variada; en cuanto a la organización no se atienen con frecuencia a la línea jerárquica.

- No hablan de planificación, coordinación. organización o control sino de todo tipo de temas.

- Hacen muchas preguntas en las conversaciones.

- Las conversaciones contienen numerosas bromas y referencias a asuntos extra trabajo.

- Con frecuencia reaccionan a iniciativas de otros. Gran parte de su día típico no es planificado o a la planificación original se suma la dedicación de tiempo a gran cantidad de tiempo a temas no incluidos en la agenda oficial.

- Trabajan largas jornadas (Garrido J.A., 1993).

Los estudios conducidos por Henry Mintzberg, profesor de la Universidad McGill de Canadá indican entre otros aspectos, que los ejecutivos exitosos manejan el problema de la información de modo muy distinto al que marca el modelo gerencial tradicional. Así plantea que el gerente busca constantemente información relacionada con el desempeño de la unidad y su entorno y comunica información valiosa (McLeod R., 2000).

El gerente de la sociedad del siglo XXI, desarrollará sus competencias gerenciales bajo la paradoja de la incertidumbre y las oportunidades de grandes libertades de pensamiento, amplitud de información y conocimientos, que le darán nuevas dimensiones de responsabilidades, de saber y de poder. Por tanto, la gerencia que se requiere hoy es aquella basada por un lado, en el conocimiento, pues éste va ser la plataforma para diseñar la mejor estrategia para la empresa, y por el otro, en la tecnología de información y comunicación, como elemento clave para desarrollar sistemas que permitan mejorar los procesos de transferencia de esos conocimientos (Montilla M., 2008). 
Se está en los umbrales de intentos de pasar del campo de "la gerencia fácil" operando en ciertas condiciones de estabilidad y competitividad limitada, a la gerencia que debe encarar contextos en continuos cambios y una competitividad agresiva y creciente (Santiesteban Amat M., 2011).

Una serie de trabajos proponen desarrollar con complejidad la orientación "feed forward". Debe descartarse el estilo gerencial propio del modelo tradicional, en donde el gerente es entrenado para hacer previsiones sobre el futuro a partir de extrapolar el pasado. El "feed forward" sugiere ajustar las decisiones presentes, no a partir del pasado, sino de los futuros previsibles. (Kliksber B., 1991).

Se requiere para encarar la complejidad, cambiar todo el esquema mental con que trabaja la gerencia en el modelo tradicional:

- El gerente debe dejar de leer la realidad desde un punto de vista único. Debe acostumbrarse a pensar proyectando mentalmente varios escenarios posibles simultáneos.

- En el modelo tradicional trata de llegar rápidamente a decisiones en las que se cierra. Deberá en lugar de ello permanecer abierto, suspendiendo juicios hasta que emerja una visión comprensiva de la situación.

- El gerente de las décadas anteriores, cuando hay diferencia de opiniones, trata de presionar a los otros a que se amolden a su punto de vista. El nuevo debe capitalizar las otras opiniones, porque la realidad compleja y ambigua, puede entenderse mejor si se aumentan los ángulos de lectura.

Están en desarrollo nuevas metodologías que aumentan la capacidad de "pensar" y "analizar" del gerente. Entre ellas el portafolio planning, los juegos de escenarios, los think thanks. La nueva ola de tecnologías de decisión: mapeos cognoscitivos, mapas de decisiones, simulaciones computarizadas. Las simulaciones pueden mejorarse mucho con los desarrollos en el campo de la inteligencia artificial. Los modelos de simulación usuales han resultado limitados frente a los problemas que plantea la realidad que desafían la simplicidad de las estructuras numéricas y las ecuaciones. La inteligencia artificial trae códigos que permiten el uso de datos simbólicos, estrategias de investigación para problemas discontinuos y procura replicar el proceso de decisión humana (Garrido J.A., 1993). 
Preparar gerentes para este tipo de capacidades requerirá no solo variar radicalmente los currículos tradicionales de formación, sino el enfoque pedagógico básico con que se encara el proceso de aprendizaje.

Junto a proporcionar un amplio bagaje cognoscitivo enmarcado en el nuevo paradigma gerencial en construcción, será imprescindible que dicho proceso tenga extensos espacios para la experimentación en el campo mismo de las dificultades que implica el desempeño gerencial. Algunas de las habilidades requeridas se desenvolverán sobre todo aprendiendo de la experiencia. (Kliksber B., 1991).

La posibilidad de contar con gerentes eficientes está ligada a los esfuerzos por dar el salto tecnológico, informacional, comunicacional y de aprendizaje. Se requieren para ello transformaciones muy profundas en los modelos y hábitos tradicionales en gerencia.

Se precisa, además, una gerencia imaginativa que pueda impulsar y liderar la reconversión de la misma. Se necesita gerencia de primera calidad para llevar adelante con la mayor eficiencia todos los cambios que se necesitan en las organizaciones.

La gerencia necesaria es gerencia de avanzada. Gerencia capaz de trabajar en un mundo interrelacionado más que nunca en la historia, en cambio tecnológico vertiginoso, en contextos tumultuosos, en medio del incremento continuo de la complejidad. (Santiesteban Amat M., 2011)

El modelo gerencial del Pentágono Intangible, propone cinco competencias que deben destacar al gerente son:

a) Empresario, para emprender nuevos caminos;

b) Educador, para fomentar el aprendizaje en su organización;

c) Consejero, para convertirse en el acompañante y amigo especial de quienes conforman su equipo de trabajo;

d) Comunicador, para edificar la comunidad de pensamientos; $y$

e) Negociador, para poder llegar a acuerdos excelentes que favorezcan a su organización. 
El paradigma de la gerencia tradicional comienza a desvanecerse y con ello el sistema de ideas que lo soportan. Hoy la gerencia debe estar enmarcada dentro de las nuevas realidades y tendencias infocomunicacionales. Lo que marcará la diferencia entre un gerente tradicional y el gerente que requiera la sociedad del siglo XXI será indiscutiblemente su forma de aproximarse a la información y el conocimiento: para los primeros seguirán siendo herramientas para producir beneficios económicos, para los segundos algo superior, más asociado a la comunicación y al entendimiento humano y social (Montilla M., 2008).

Según Marrero (2001), los 7 pecados capitales de los gerentes que fracasan son:

1. Falta de empuje. Presionar es el trabajo más duro del directivo, para ello hace falta tener energía, vigor, fuerza mental, ganas pegadas. Es más fácil domar a un salvaje que dar vida a un cadáver. Fracasan más los ejecutivos por cadáveres que por fanáticos. No fracasan por probar y errar, fracasan por no probar, por no presionar.

2. Falta de imaginación. Falta de habilidad para prever lo que queda por hacer, son mejores obedeciendo que conduciendo, no salen al encuentro de los problemas, no prevén los errores de su personal, cuando ocurren buscan culpables en lugar de haberlos formados adecuadamente, viven en constante crisis y sobresalto, conducen sin visibilidad.

3. Falta de estilos compatibles. Cuando cada ejecutivo viene de su casa con su estilo y estos no son compatibles, estalla el departamentismo, la incapacidad de comunicarse, la falta de detalles, la falta de agilidad, son partes de este estilo que nada tiene que ver con la calidad total.

4. La incapacidad de comunicarse. El hacerse entender es el arma básica de cualquier ejecutivo para que le sigan. Pero para ello debe dominar la técnica de la comunicación.

5. La incapacidad de sumar. El creer que los galones de mando dan de por si la razón, lleva a la apatía de los colaboradores. Hay que exhibir grandes dotes de saber escuchar, es decir, de sumar ideas, opiniones para que los colaboradores se vean y se sientan útiles.

6. La incapacidad de dimitir. Cuantas veces oímos que un ejecutivo no está de acuerdo con alguna decisión tomada por sus superiores, pero calla y critica por detrás. Cuando uno no está 
de acuerdo debe comunicarlo con suficientes argumentos para intentar persuadir, si no lo consigue tiene dos opciones, asumir la decisión y colaborar a su éxito o dimitir.

7. La incapacidad para satisfacer necesidades. Los directivos se olvidan que fueron creados única y exclusivamente para satisfacer las necesidades de los clientes, para conseguir que estos repitan y para conseguir que los otros clientes, los empleados, repitan en eficacia.

En suma, la reconstrucción de las representaciones mentales de los gerentes de este siglo, mediante nuevas concepciones, es imprescindible para intervenir en la realidad de los fenómenos organizacionales. Gerentes innovadores han de inventar sus propios rumbos (...) con nuevas estrategias de pensamiento y acabando con recetas metódicas preestablecidas que frenan la creatividad y la imaginación (...) Se requiere de gerentes con mente flexible, es decir, aquella que no está fija en un punto ni se desliza por cualquier parte sin rumbo, sino que posee una dirección renovable (...) La fuerza del pensamiento flexible radica en que, a pesar de la resistencia y los obstáculos, permite que el gerente se invente a sí mismo (Pérez M.A., 2014).

\section{Conclusiones}

El futuro del desempeño exitoso de los recursos humanos y, en especial de los gerentes, tendrá que ver cada vez más con la acepción holística implicada en las competencias, entendidas así al incluir no sólo conocimientos y habilidades para hacer el trabajo (saber hacer), sino también actitudes, valores y rasgos personales vinculados al buen desempeño en el mismo (querer hacer).

Existen cuestiones, más allá de las funciones gerenciales, que el gerente debe incorporar para obtener mejores resultados, éstas se concentran en la información, la comunicación, la formación y el enfrentamiento dinámico a la complejidad con mente flexible e innovadora.

\section{Referencias bibliográficas}

Drucker D. (1954). The Practice of Management. Nueva York: Harper and Row.

Garrido J.A. (1993). Estrategia empresarial ante el caos. Madrid.

Hersey Paul, K. H. (1998). Administración del comportamiento organizacional. Liderazgo situacional. Prentice Hall. 
Hodge B.J., G. L. (1998). Teoría de la organización. Un enfoque estratégico. Madrid:

PRENTICE HALL IBERIA.

KLIKSBER B. (1991). COMO SERA LA GERENCIA EN LA DECADA DEL 90?

Koontz H., C. O. (1959). Principles of Management. Nueva York: McGraw-Hill.

McLeod R. (2000). Sistemas de información gerencial.

Montilla M., M. R. (2008). Competencias clave del gerente en el contexto de la sociedad de la información. Telematique, Volumen 7 - Número 1.

Pérez M.A., A. C. I. (2014). Formación gerencial y epistemología. Formación Gerencial, Año 13 $\mathrm{N}^{\mathrm{o}} 1$.

Robbins, S. (1992). Administración.

Santiesteban Amat M. (2011). Marketing, relaciones públicas, gerencia y NTIC a las puertas del siglo XXI.

Tannenbaum R., I. R. (1959). Leadership and organization: A behavioral science approach. Nueva York, McGraw-Hill, 1959. Nueva York: McGraw-Hill.

Terry, G. R. (1960). Principles of Management.

USACA. (s/f). Gerencia empresarial y global. Obtenido de http://docentes.usaca.edu.co/marmol/html/gerencia.html. 